\title{
Clinical impact of anti-thymocyte globulin on survival and graft-versus-host disease in patients undergoing human leukocyte antigen mismatched allogeneic stem cell transplantation
}

Taeyun Kim ${ }^{1, *}$, Yunsuk Choi ${ }^{2,}{ }^{*}$, Je-Hwan Lee ${ }^{3}$, Silvia Park ${ }^{4}$, Jae-Sook Ahn ${ }^{5}$, Joon-Ho Moon ${ }^{6}$, Ho-Jin Shin ${ }^{7}$, Won Sik Lee ${ }^{8}$, Dajung Kim ${ }^{1}$, and Ho Sup Lee ${ }^{1}$

\begin{abstract}
${ }^{1}$ Department of Internal Medicine, Kosin University Gospel Hospital, Busan; ${ }^{2}$ Department of Hematology and Oncology, Ulsan University Hospital, University of Ulsan College of Medicine, Ulsan; ${ }^{3}$ Department of Hematology, Asan Medical Center, University of Ulsan College of Medicine, Seoul; ${ }^{4}$ Division of Hematology-Oncology, Samsung Medical Center, Sungkyunkwan University School of Medicine, Seoul; ${ }^{5}$ Department of Internal Medicine, Chonnam National University Hwasun Hospital, Hwasun; ${ }^{6}$ Department of Hematology, Kyungpook National University Hospital, Daegu; ${ }^{7}$ Department of Internal Medicine, Pusan National University Hospital, Busan; ${ }^{8}$ Department of Internal Medicine, Inje University Busan Paik Hospital, Busan, Korea
\end{abstract}

Received: December 27, 2017

Revised : March 2, 2018

Accepted: May 4, 2018

\section{Correspondence to}

Ho Sup Lee, M.D.

Department of Internal Medicine, Kosin University Gospel Hospital, 262 Gamcheon-ro, Seo-gu, Busan 49267, Korea

Tel: +82-51-990-5820

Fax: +82-51-990-5820

E-mail: hs52silver@gmail.com

*'These authors contributed equally to this work.

Background/Aims: Rabbit anti-thymocyte globulin (ATG) is usually incorporated in hematopoietic stem cell transplantation (HSCT) to reduce the incidence of graft-versus-host disease (GVHD). This study aimed to find optimal ATG doses in patients undergoing human leukocyte antigen (HLA)-mismatched allogeneic HSCT.

Methods: We retrospectively collected medical records from 352 consecutive patients with acute myeloid leukemia $(\mathrm{n}=214)$, acute lymphoblastic leukemia $(\mathrm{n}=$ 62), or myelodysplastic syndrome $(n=76)$ in eight centers of Korea between 2005 and 2015. All patients received busulfan-based conditioning without total body irradiation (TBI) and received stem cells from HLA-mismatched donors.

Results: In the current study, 5-year overall survival rates of patients receiving low to medium doses of ATG (2.5 to $7.5 \mathrm{mg} / \mathrm{kg}$ ) were higher than those receiving other doses of ATG (hazard ratio [HR], 0.528; 95\% confidence interval [CI], 0.311 to $0.897 ; p=0.018$ ). The incidence rates of extensive chronic GVHD (ecGVHD) after administration of low to medium doses of ATG were lower than those after other doses of ATG (HR, $0.447 ; 95 \%$ CI, 0.224 ton $0.889 ; p=0.022)$.

Conclusions: The low to medium doses of ATG may be associated with improving survival outcomes and reducing incidence of ecGVHD without enhancing the chances of relapse in patients with acute leukemia or myelodysplastic syndrome undergoing non-TBI-based HLA-mismatched allogeneic HSCT.

Keywords: Antithymocyte globulin; Graft vs host disease; Survival; Human leukocyte antigen mismatch; Allogeneic hematopoietic stem cell transplantation

\section{INTRODUCTION}

Allogeneic hematopoietic stem cell transplantation (HSCT) is a curative modality for treating hematologic malignancies [1]. Previously, when histo- compatibility antigens were difficult to identify, allogeneic HSCT was fatal [2]. HSCT from mismatched unrelated or haploidentical family donors usually increased the risk of graft-versus-host disease (GVHD) and transplant-related 
mortality (TRM) [3]. Anti-thymocyte globulin (ATG) is used to prevent GVHD and has improved survival rates by decreasing the incidence of GVHD [4]. Over the past several decades, the use of ATG has contributed significantly in improving the outcomes of alternative donor transplantation [5]. ATG, however, may also contribute to the risk of relapse and infections, as well as of early TRM [3]. Higher doses (8 mg/kg or more) of ATG can increase the risk of relapse and decrease relapse free survival after reduced intensity conditioning (RIC) HSCT with unrelated donors [6]. There have been some retrospective trials to address the true effects of ATG on the outcome and to determine its optimal dose on the basis of human leukocyte antigen (HLA) disparity $[7,8]$. A recent study reported that ATG at a dose lower than $6 \mathrm{mg} / \mathrm{kg}$ was sufficient to prevent GVHD [9]. Another study recommended 4.5 to 5 and $7.5 \mathrm{mg} / \mathrm{kg}$ ATG under identical sibling donor and unrelated donor settings, respectively [10]. Therefore, we aimed to investigate the effects of different ATG doses on survival outcome and acute GVHD (aGVHD) and chronic GVHD (cGVHD) incidence in HLA-mismatched HSCT receiving busulfan-based conditioning regimen.

\section{METHODS}

\section{Patients}

This study was performed retrospectively in eight hospitals of South Korea from 2005 to 2015. In total, 352 patients suffering from acute leukemia or myelodysplastic syndrome (MDS) who underwent HSCT from HLA-mismatched unrelated or haploidentical family donors were included in the study. All patients received busulfan-based conditioning regimen, including busulfan plus cyclophosphamide or busulfan plus fludarabine. Patients who had been treated using total body irradiation (TBI)-based conditioning regimens were not included in this study. The pre-transplant disease status was divided into first or second complete remission (CR1 or CR2), refractory or relapsed, and untreated status. The definition of CR followed the recommended criteria [11]. To evaluate the degree of HLA matching, HLA A, B, C, and $D R$ were identified using polymerase chain reaction with sequence specific primer followed by high-resolution typing, and the degree of HLA matching was clas- sified as 1 antigen mismatch, 2 antigen mismatches, 3 antigen mismatches, and 4 antigen mismatches. Stem cell sources, such as bone marrow or peripheral blood were identified and $\mathrm{CD}_{34}+$ cell counts $\left(\times 10^{6} / \mathrm{kg}\right)$ were measured to evaluate the amount of stem cells. This study was approved by Institutional Review Board (IRB) of Kosin University Gospel Hospital (approval number 201809011) and the IRBs granted a waiver of informed consent because this study was a retrospective analysis that involved no more than minimal risk for patients.

\section{Transplantation procedure}

Busulfan and fludarabine (BuFlu) or busulfan and cyclophosphamide (BuCy) were used as conditioning regimens, including Bu4Cy (busulfan $[3.2 \mathrm{mg} / \mathrm{kg}]$ for 4 days along with cyclophosphamide [ $60 \mathrm{mg} / \mathrm{kg}$ ] for 2 days), Bu4Flu (busulfan $[3.2 \mathrm{mg} / \mathrm{kg}]$ for 4 days along with fludarabine [30 mg/kg] for 6 days), Bu3Flu (busulfan [3.2 mg/kg] for 3 days along with fludarabine [30 mg/kg] for 6 days), Bu2Flu (busulfan [3.2 mg/kg] for 2 days along with fludarabine [30 mg/kg] for 6 days), and BurFlu (busulfan [3.2 $\mathrm{mg} / \mathrm{kg}$ ] for 1 day along with fludarabine [30 $\mathrm{mg} / \mathrm{kg}$ ] for 6 days). Bu4Cy and Bu4Flu were defined as myeloablative conditioning (MAC), and Bu3Flu, Bu2Flu, and BurFlu were defined as RIC. Rabbit ATG (thymoglobulin, Sanofi-Aventis, Cambridge, MA, USA) was administered to all patients at various dosages to prevent GVHD. We classified patients who did not receive ATG as no ATG group (o mg/kg) and those who received 2.5 to $7.5 \mathrm{mg} / \mathrm{kg}$ (less than $8 \mathrm{mg} / \mathrm{kg}$ ) as low to medium ATG group. Patients who received 9 to $12 \mathrm{mg} / \mathrm{kg}$ ATG were classified as high ATG group. ATG was given as an equally divided dose for 2 or 3 days from day -3 . All patients received calcineurin inhibitors, including cyclosporine or tacrolimus with or without short-term methotrexate, as immunosuppressants to prevent GVHD.

\section{Statistical analysis}

Survival probabilities were estimated using the Kaplan-Meier method and differences in survival distributions were compared using the log-rank test. Overall survival (OS) rates were calculated from the time of HSCT to the date of death or last follow-up. Event-free survival (EFS) rates were defined from the time of HSCT to the date of relapse, progression, graft failure, donor lymphocyte infusion, or death from any cause. To identi- 
fy factors affecting OS and EFS, the log rank test and Cox proportional hazard model were used in univariate and multivariate analysis, respectively. ATG dose and other variables having $p<0.05$ in the univariate analysis were included for multivariate analysis. We included ATG doses as a variable in multivariate analysis to validate the clinical impact of ATG dose on survival outcomes and GVHD incidence, although there were no significant differences with respect to ATG doses in univariate analysis. Consensus grading criteria was used to assess the degree of aGVHD [12]. The cumulative incidence rate (CIR) of aGVHD was calculated through day 100. A cGVHD was assessed using the traditional grading criteria [13]. For all analyses, the $p$ values were two-sided, and $p<0.05$ was considered statistically significant. The statistical analyses were performed using SPSS version 24.0 software (IBM Corp., Armonk, NY, USA).

The CIR of relapse and GVHD were generated using confidence interval (CI) estimates adjusting for competing risks and compared by Gray's method. For the analysis of CIR of relapse, patients who were alive without relapse were censored.

\section{RESULTS}

\section{Patient characteristics}

This study enrolled patients with acute leukemia or MDS who underwent HLA-mismatched HSCT. Their baseline characteristics are summarized in Table 1 . The median age was 46 years (range, 15 to 75 ). Two hundred and nine patients were diagnosed with acute myeloid leukemia (AML, 59.4\%), 62 patients with acute lymphoblastic leukemia (ALL, 17.6\%), five patients had mixed phenotype acute leukemia (MPAL, 1.4\%), and 76 patients were diagnosed with MDS (21.6\%). Of these 352 patients, 172 patients (48.8\%) were confirmed as CR1 status, 29 patients $(8.2 \%)$ as CR2 or subsequent remission, 55 patients (15.6\%) as refractory, 34 (9.7\%) as relapse, and 62 patients $(17.6 \%)$ as untreated before HSCT. Of all recruited patients, 264 patients (75.0\%) received RIC while 88 patients $(25.0 \%)$ received MAC for conditioning. One hundred and seventy-one patients (48.6\%) received stem cells from haploidentical donors and 181 patients (51.4\%) from HLA-mismatched unrelated donors. HLA 1 locus mismatch was detected in 107 patients (30.4\%), 2 mis-
Table 1. Baseline characteristics

\begin{tabular}{|c|c|}
\hline Parameter & All patients $(\mathrm{n}=352)$ \\
\hline Age, yr & $46(15-75)$ \\
\hline \multicolumn{2}{|l|}{ Sex } \\
\hline Male & $210(59 \cdot 7)$ \\
\hline Female & $142(40.3)$ \\
\hline \multicolumn{2}{|l|}{ Diagnosis } \\
\hline AML & $209(59.4)$ \\
\hline ALL & $62(17.6)$ \\
\hline MPAL & $5(1.4)$ \\
\hline MDS & $76(21.6)$ \\
\hline \multicolumn{2}{|l|}{ Disease status } \\
\hline $\mathrm{CR} 1$ & $172(48.8)$ \\
\hline$\geq \mathrm{CR} 2$ & $29(8.2)$ \\
\hline Refractory/Relapse & $89(25 \cdot 4)$ \\
\hline Untreated & $62(17.6)$ \\
\hline \multicolumn{2}{|l|}{ Intensity of conditioning } \\
\hline MAC & $88(25.0)$ \\
\hline RIC & $264(75.0)$ \\
\hline \multicolumn{2}{|l|}{ ATG dose, $\mathrm{mg} / \mathrm{kg}$} \\
\hline $\mathrm{O}$ & $43(12.2)$ \\
\hline $2.5-7.5$ & $60(17.0)$ \\
\hline 9.0 & $192(54.5)$ \\
\hline 12.0 & $57(16.2)$ \\
\hline \multicolumn{2}{|l|}{ Type of donors } \\
\hline Related & $171(48.6)$ \\
\hline Unrelated & $181(51.4)$ \\
\hline \multicolumn{2}{|c|}{ HLA match (A,B,C, and DR), allelic level } \\
\hline 1 mismatch & $107(30.4)$ \\
\hline 2 mismatch & $53(15 \cdot 1)$ \\
\hline 3 mismatch & $39(11.1)$ \\
\hline 4 mismatch & $153(43 \cdot 5)$ \\
\hline \multicolumn{2}{|l|}{ Stem cell source } \\
\hline Bone marrow & $93(26.4)$ \\
\hline Peripheral blood stem cell & $259(73.6)$ \\
\hline $\mathrm{CD}_{34}+$ cell dose, $\times 10^{6} / \mathrm{kg}$ & $6.15(0.230-38.33)$ \\
\hline Donor age, yr & $30(3-70)$ \\
\hline \multicolumn{2}{|l|}{ Donor sex } \\
\hline Male & $235(66.8)$ \\
\hline Female & $112(31.8)$ \\
\hline
\end{tabular}

Values are presented as median (range) or number (\%). AML, acute myeloid leukemia; ALL, acute lymphoblastic leukemia; MPAL, mixed phenotype acute leukemia; MDS, myelodysplastic syndrome; CR, complete remission; MAC, myeloablative conditioning; RIC, reduced intensity conditioning; ATG, anti-thymocyte globulin; HLA, human leukocyte antigen. 
matches in 53 patients (15.1\%), 3 mismatches in 39 patients (11.1\%), and 4 mismatches in 153 patients (43.5\%). Among the patients who received stem cells from mismatched family donors, 161 patients (94.2\%) had HLA 3 or 4 mismatches, and among those who received from unrelated donors, 31 patients (17.1\%) had HLA 3 or 4 mismatches. Forty-three patients (12.2\%) did not receive ATG during transplantation, 60 patients (17.0\%) were treated with 2.5 to $7.5 \mathrm{mg} / \mathrm{kg}$ of ATG, 192 patients (54.5\%) with $9.0 \mathrm{mg} / \mathrm{kg}$ of ATG, and 57 patients (16.2\%) with 12.0 $\mathrm{mg} / \mathrm{kg}$ of ATG.

\section{Prognostic factors for OS, EFS, and relapse}

In univariate analysis, there were significant differences in 5-year OS rates according to disease status $39.1 \%$ in $\mathrm{CR} 1,54.0 \%$ in $\geq \mathrm{CR} 2,11.5 \%$ in relapse/refractory, and $38.8 \%$ in untreated status, $p<0.001)$. In addition, conditioning intensity had significant impact on OS $(18.2 \%$ in MAC group vs. $36.3 \%$ in RIC group, $p=0.026$ ). However, there was no significant difference in 5-year OS according to the other clinical factors: sex (male 30.3\% and female $34.8 \%, p=0.842)$, age $(<50$ years $37.8 \%$ and $\geq 50$ years $24.3 \%, p=0.096$ ), diagnosis (AML 30.1\%, ALL 34.1\%, MPAL 53.3\%, and MDS 40.1\%, $p=0.829$ ), HLA mismatch (1 mismatch 30.8\%, 2 mismatches $44.8 \%, 3$ mismatches $23.2 \%$, and 4 mismatches $32.9 \%, p=0.326$ ), stem cell source (bone marrow $34.8 \%$ and peripheral blood $33.5 \%, p=0.238), \mathrm{CD}_{34}+$ cell dose $\left(<2.0 \times 10^{6} / \mathrm{kg} 51.4 \%\right.$ and $\geq 2.0 \times 10^{6} / \mathrm{kg} 32.1 \%, p=0.40$ ), and median donor age $(<30$ years $36.0 \%$, 30 to 49 years $32.3 \%$, and $\geq 50$ years $20.1 \%, p=0.067)$. There were significant differences in the 5-year EFS based on disease status (38.7\% for CR1, $44.5 \%$ for $\geq$ CR2, $9.4 \%$ for relapse/refractory, and 33.6\% for untreated status, $p<0.001)$ and median donor age $(<$ 30 years $37.0 \%$, 30 to 49 years $26.3 \%$, and $\geq 50$ years $17.8 \%$, $p=0.010)$. However, there was no significant difference in EFS on the basis of other clinical factors, including sex (male $28.9 \%$ and female $31.2 \%, p=0.97$ ), patient's age ( $<50$ years $34.6 \%$ and $\geq 50$ years $23.6 \%, p=0.364$ ), diagnosis (AML 28.1\%, ALL 37.3\%, MPAL 30.0\%, and MDS $35.2 \%, p=0.299$ ), conditioning intensity (MAC 25.3\% and RIC $32.6 \%, p=0.132)$, HLA mismatch (1 mismatch $32.5 \%$, 2 mismatches $38.5 \%, 3$ mismatches $17.6 \%$, and 4 mismatches $28.1 \%, p=0.174$ ), stem cell source (bone marrow $29.8 \%$ and peripheral blood $32.3 \%, p=0.168)$, and $\mathrm{CD}_{34}+$ cell dose $\left(<2.0 \times 10^{6} / \mathrm{kg} 39.3 \%\right.$ and $\geq 2.0 \times 10^{6} / \mathrm{kg}$
$30.5 \%, p=0.886)$. The 5 -year CIR of relapse, relapsed or refractory disease status at the time of HSCT was significantly associated with higher relapse compared to CR or untreated status $(83.2 \%$ in relapse/refractory vs. $39.3 \%$ in $\mathrm{CR} 1,48.2 \%$ in $\geq \mathrm{CR} 2$, and $30.4 \%$ in untreated status, $p$ $<0.001$ ). However, there was no significant association between CIR of relapse and other clinical factors, such as sex (male $51.4 \%$ and female $48.9 \%, p=0.906$ ), age ( $<50$ years $49.3 \%$ and $\geq 50$ years $49.4 \%, p=0.868)$, conditioning intensity (MAC 50.4\% and RIC 49.3\%, $p=0.958$ ), stem cell source (bone marrow $48.6 \%$ and peripheral blood $48.6 \%$, $p=0.402), C_{34}+$ cell dose $\left(<2.0 \times 10^{6} / \mathrm{kg} 50.9 \%\right.$ and $\geq 2.0$ $\left.\times 10^{6} / \mathrm{kg} 49.3 \%, p=0.532\right)$, and median donor age $(<30$ years $46.9 \%$, 30 to 49 years $51.4 \%$, and $\geq 50$ years $55.3 \%, p$ $=0.614)$. However, three ATG groups did not show significant differences in their 5-year OS (30.7\% in no ATG group, $46.2 \%$ in low to medium dose ATG group, and $32.2 \%$ in high dose ATG group, $p=0.615$ ) and, 5 -year EFS (37.6\% in no ATG group, $41.5 \%$ in low to medium dose ATG group, and $29.0 \%$ in high dose ATG group, respectively; $p=0.289$ ). In addition, there was also no significant difference in 5-year CIR of relapse among the three ATG groups (39.9\% in no ATG group, $42.1 \%$ in low to medium dose ATG group, and 51.4\% in high dose ATG group, respectively; $p=0.289$ ).

\section{Prognostic factors for grade 2 to 4 aGVHD and ex- tensive chronic GVHD}

In univariate analysis, three ATG groups did not significantly affect the CIR of grade 2 to 4 aGVHD. However, the low to medium ATG group tended to show lower incidence rate of grade 2 to 4 aGVHD than those of other two groups (29.6\% in low to medium ATG vs. $46.3 \%$ in no ATG group and $34.5 \%$ in high ATG group, $p=0.083$ ) although the difference was not statistically significant. Age and conditioning intensity significantly affected grade 2 to 4 aGVHD incidence; age (<50 years $38.6 \%$ and $\geq 50$ years $29.9 \%, p=0.018)$ and conditioning intensity (MAC 48.4\% and RIC 30.8\%, $p=0.003$ ). However, there was no significant association between grade 2 to 4 aGVHD incidence and other clinical factors, including $\operatorname{sex}(35.2 \%$ in male and $34.8 \%$ in female, $p=0.907$ ), disease status (31.6\% in CR1, 21.4\% in $\geq \mathrm{CR} 2,47.4 \%$ in untreated, and $37.9 \%$ in refractory/relapse status, $p=0.05$ ), stem cell source $(42.3 \%$ for bone marrow and $32.6 \%$ for peripheral blood, $p=0.211), C_{34}+$ cell dose $(35.7 \%$ in $<2.0 \times$ 
Table 2. Multivariate analysis of the 5-year OS rates, the 5-year EFS rates, and the 5-year cumulative incidence rates of relapse in patients with acute leukemia and myelodysplastic syndrome

\begin{tabular}{|c|c|c|c|c|c|c|}
\hline \multirow[t]{2}{*}{ Parameter } & \multicolumn{2}{|l|}{5 -yr OS } & \multicolumn{2}{|l|}{ 5-yr EFS } & \multicolumn{2}{|c|}{$\begin{array}{c}\text { 5-yr cumulative incidence of } \\
\text { relapse }\end{array}$} \\
\hline & $\mathrm{HR}(95 \% \mathrm{CI})$ & $p$ value & $\mathrm{HR}(95 \% \mathrm{CI})$ & $p$ value & $\operatorname{HR}(95 \% \mathrm{CI})$ & $p$ value \\
\hline \multicolumn{7}{|l|}{ Total ATG dose, mg/kg } \\
\hline o & 1 & & 1 & & 1 & \\
\hline $2.5-7.5$ & $0.528(0.311-0.897)$ & 0.018 & $0.792(0.496-1.263)$ & 0.327 & $0.580(0.300-1.122)$ & 0.105 \\
\hline $9-12$ & $0.716\left(0.465^{-1.101}\right)$ & 0.128 & $0.902(0.612-1.329)$ & 0.602 & $0.936\left(0.575^{-1.521}\right)$ & 0.789 \\
\hline \multicolumn{7}{|l|}{ Disease status } \\
\hline $\mathrm{CR} 1$ & 1 & & 1 & & 1 & \\
\hline$\geq \mathrm{CR} 2$ & $0.718(0.379-1.360)$ & 0.309 & $0.997(0.580-1.713)$ & 0.992 & $1.152(0.614-2.161)$ & 0.001 \\
\hline Refractory/Relapse & $2.903(2.101-4.009)$ & $<0.001$ & $2.803(2.036-3.858)$ & $<0.001$ & $1.726(0.787-3.787)$ & 0.038 \\
\hline Untreated & $1.290(0.849-1.960)$ & 0.232 & $1.275(0.862-1.886)$ & 0.224 & $4.964(2.692-9.152)$ & $<0.001$ \\
\hline \multicolumn{7}{|l|}{ Conditioning intensity } \\
\hline MAC & 1 & & & & & \\
\hline RIC & $2.051(1.404-2.996)$ & $<0.001$ & & & & \\
\hline \multicolumn{7}{|l|}{ Age of donor, yr } \\
\hline$<30$ & & & 1 & & & \\
\hline $30-49$ & & & $1.293(0.975-1.716)$ & 0.075 & & \\
\hline$\geq 50$ & & & $1.328(0.807-2.185)$ & 0.264 & & \\
\hline
\end{tabular}

OS, overall survival; EFS, event-free survival; HR, hazards ratio; CI, confidence interval; ATG, anti-thymocyte globulin; CR, complete remission; MAC, myeloablative conditioning; RIC, reduced intensity conditioning.

$10^{6} / \mathrm{kg}$ and $35.2 \%$ in $\left.\geq 2.0 \times 10^{6} / \mathrm{kg}, p=0.76\right)$, and median donor age $(33.7 \%$ in $<30$ years, $33.6 \%$ in 30 to 49 years, and $46.8 \%$ in $\geq 50$ years, $p=0.316$ ). However, the CIR of extensive chronic GVHD (ecGVHD) significantly varied among ATG groups $(62.1 \%$ in no ATG, $27.1 \%$ in low to medium ATG, and $36.1 \%$ in high ATG group, $p=0.012$ ). With respect to the stem cell source, patients receiving peripheral blood stem cells showed significantly higher ecGVHD rate compared to those receiving bone marrow stem cell (44.2\% vs. $22.1 \%$, respectively; $p<0.001$ ). However, there was no significant association between ecGVHD incidence and other factors, such as age $(42.1 \%$ in $<50$ years and $34.8 \%$ in $\geq 50$ years, $p=0.42$ ), disease status $(35.5 \%$ in $\mathrm{CR} 1,37.7 \%$ in $\geq \mathrm{CR} 2,37.9 \%$ in untreated, and $69.0 \%$ in refractory/relapse status, $p=0.58)$, conditioning intensity (70.5\% in MAC and $35.5 \%$ in RIC, $p=0.557$ ), HLA mismatch $(54.2 \%$ in 1 mismatch, $23.8 \%$ in 2 mismatches, $40.7 \%$ in 3 mismatches, and $31.9 \%$ in 4 mismatches, $p=$ $0.469), C_{34}+$ cell dose $\left(54.1 \%\right.$ in $<2.0 \times 10^{6} / \mathrm{kg}$, and $38.7 \%$ in $\left.\geq 2.0 \times 10^{6} / \mathrm{kg}, p=0.071\right)$, and median donor age $(34.3 \%$ in $<30$ years, $45.4 \%$ in 30 to 49 years, and $28.3 \%$ in $\geq 50$ years, $p=0.852$ ).

\section{Multivariate analysis for OS, EFS, relapse, aGVHD, and ecGVHD}

The factors affecting the 5-year OS and 5-year EFS in multivariate analysis are shown in Table 2. The low to medium ATG group showed significantly longer survival compared with other ATG group (hazard ratio [HR], 0.528; 95\% CI, 0.311 to 0.897 ; $p=0.018$ ) (Fig. 1A). Moreover, the 5-year OS of patients conditioned with RIC and with disease status of refractory/relapse was lower than that of patients conditioned with MAC and with disease status of $\mathrm{CR} 1$, respectively ([HR, 2.051; 95\% CI, 1.404 to 2.996; $p<0.001$ ] and [HR, 2.903; 95\% CI, 2.101 to 4.009; $p$ $<0.001])$. The 5-year EFS of patients with disease status of refractory/relapse was significantly lower than that of patients with disease status of CR1 (HR, 2.803; 95\% CI, 2.036 to $3.858 ; p<0.001)$. But there was no significant association between the 5-year EFS with ATG dose 
A

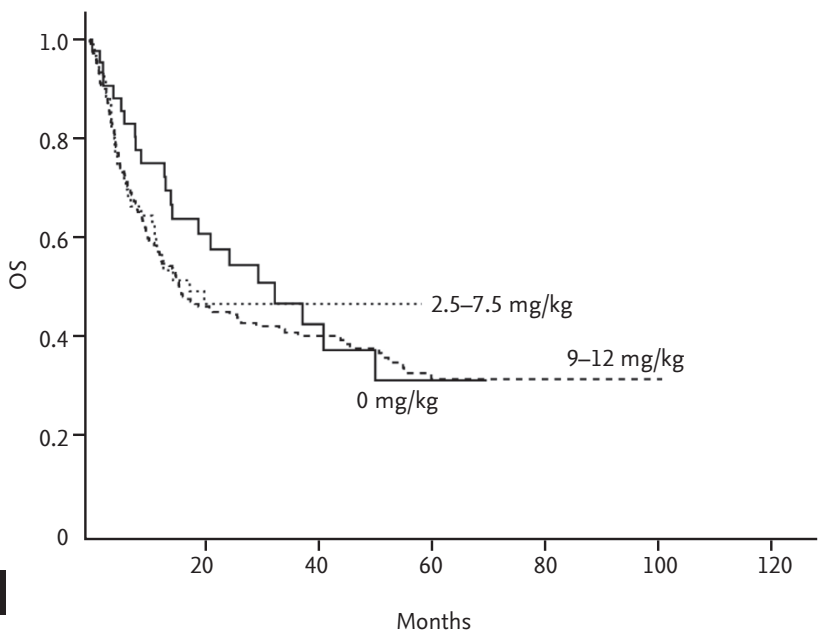

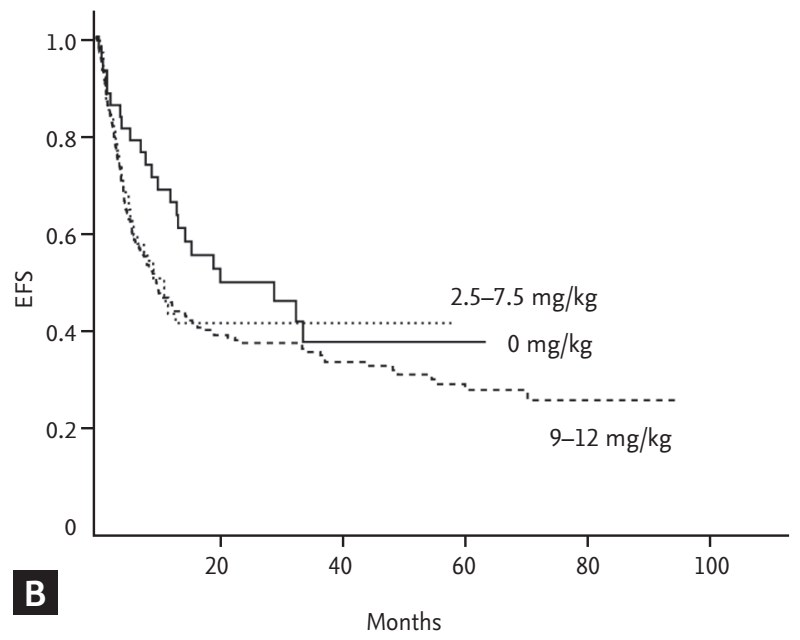

Figure 1. (A) The 5-year overall survival rates (OS) of the three anti-thymocyte globulin (ATG) groups (o, 2.5 to 7.5, and 9 to 12 $\mathrm{mg} / \mathrm{kg})$. (B) The 5-year event-free survival rates (EFS) of the three ATG groups.

Table 3. Multivariate analysis of grade 2-4 acute GVHD and extensive chronic GVHD in patients with acute leukemia and myelodysplastic syndrome

\begin{tabular}{|c|c|c|c|c|}
\hline \multirow{2}{*}{ Parameter } & \multicolumn{2}{|c|}{ Grade $2-4$ acute GVHD } & \multicolumn{2}{|c|}{ Extensive chronic GVHD } \\
\hline & $\operatorname{HR}(95 \% \mathrm{CI})$ & $p$ value & $\operatorname{HR}(95 \% \mathrm{CI})$ & $p$ value \\
\hline \multicolumn{5}{|l|}{ Total ATG dose, mg/kg } \\
\hline o & 1 & & 1 & \\
\hline $2.5-7.5$ & $0.908(0.490-1.681)$ & 0.759 & $0.447(0.224-0.889)$ & 0.022 \\
\hline $9-12$ & $0.533(0.287-0.992)$ & 0.047 & $0.678(0.442-1.038)$ & 0.074 \\
\hline \multicolumn{5}{|l|}{ Age of patient, yr } \\
\hline$<50$ & 1 & & & \\
\hline$\geq 50$ & $1.519(1.012-2.278)$ & 0.044 & & \\
\hline \multicolumn{5}{|l|}{ Conditioning intensity } \\
\hline MAC & 1 & & & \\
\hline RIC & $2.140(1.299-3.524)$ & 0.003 & & \\
\hline \multicolumn{5}{|l|}{ Stem cell source } \\
\hline Bone marrow & & & 1 & \\
\hline Peripheral blood stem cell & & & $0.887(0.572-1.375)$ & 0.591 \\
\hline
\end{tabular}

GVHD, graft-versus-host disease; HR, hazards ratio; CI, confidence interval; ATG, anti-thymocyte globulin; MAC, myeloablative conditioning; RIC, reduced intensity conditioning.

(HR, 0.792; $95 \%$ CI, 0.496 to 1.263; $p=0.327$ ) (Fig. 1B). The 5-year CIR of relapse for low to medium ATG group was higher than those of other ATG groups (HR, 0.580; $95 \%$ CI, 0.300 to 1.122; $p=0.105$ ) but the 5-year CIR of relapse of patients with disease status of CR1 was lower compared to patients with other disease statuses (Table 2). The results of multivariate analysis for CIR of GVHD are described in Table 3. The CIR of grade 2 to 4 aGVHD was significantly lower in high ATG group than that in other ATG groups (HR, 0.533; 95\% CI, 0.287 to $0.992 ; p=$ 0.047) (Fig. 2A). However, the CIR of grade 2 to 4 aGVHD was significantly higher for patients of older age ( 50 years or more) and conditioned with RIC than that of patients less than 50 years old and conditioned with MAC, respec- 

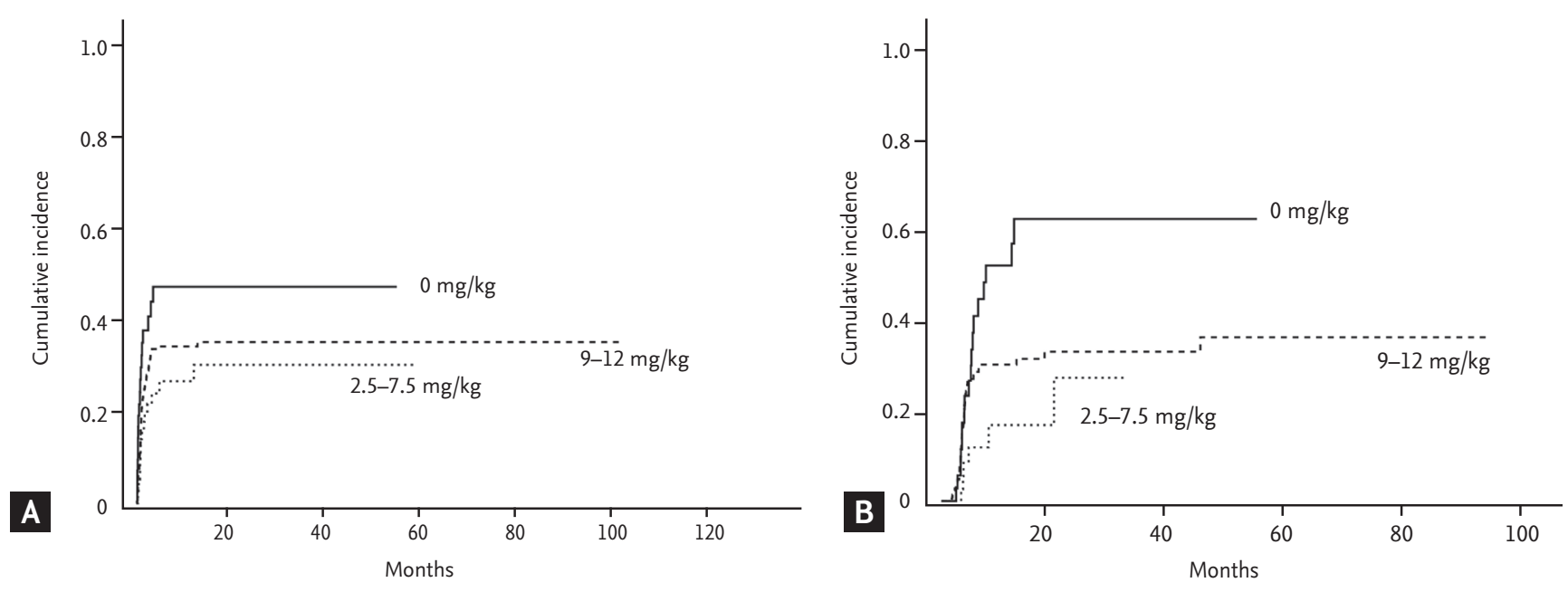

Figure 2. (A) The cumulative incidence rates (CIRs) of grade 2 to 4 acute graft-versus-host disease (GVHD) of the three anti-thymocyte globulin (ATG) groups (o, 2.5 to 7.5, and 9 to $12 \mathrm{mg} / \mathrm{kg}$ ). (B) The CIRs of extensive chronic GVHD of the three ATG groups.

tively ([HR, 1.519; 95\% CI, 1.012 to $2.278 ; p=0.044]$ and [HR, 2.140; $95 \% \mathrm{CI}, 1.299$ to $3.524 ; p=0.003])$. In addition, the CIR of ecGVHD was significantly lower in low to medium ATG group compared to that in no AGT group (HR, 0.447; 95\% CI, 0.224 to $0.889 ; p=0.022$ ) (Fig. $2 \mathrm{~B}$ ).

\section{DISCUSSION}

Our study showed that the low to medium dose of ATG (2.5 to $7.5 \mathrm{mg} / \mathrm{kg}$ ) was associated with longer survival and lower incidence rates of ecGVHD in patients who underwent HLA mismatched allogeneic HSCT. Moreover, the low to medium dose of ATG did not increase CIR of relapse after HLA mismatched allogeneic HSCT.

The cGVHD occurs in approximately 30\% to $70 \%$ of patients undergoing allogeneic HSCT of unmanipulated donor grafts and receiving a calcineurin inhibitor and antimetabolite for prophylaxis [14]. All grade aGVHD occurs in $30 \%$ to $50 \%$ of allogeneic HSCT and grade 3 to 4 GVHD occurs in 14\% [15]. There have been many trials which aimed at alleviating aGVHD and cGVHD, improving survival outcomes and reducing relapse [16-18]. The use of ATG has been known to reduce the incidence of GVHD and the severity of cGVHD through in vivo T-cell depletion [19]. However, the optimal dose of ATG with respect to prevent the GVHD is not fully defined.

In the Gruppo Italiano Trapianti Midollo Osseo (GIT-
MO) trial, two studies were conducted on the use of varying ATG doses (no ATG, $7.5 \mathrm{mg} / \mathrm{kg}$ of ATG, and $15 \mathrm{mg} / \mathrm{kg}$ of ATG) to prevent GVHD due to transplantation from unrelated donors. Use of $15 \mathrm{mg} / \mathrm{kg}$ of ATG before HSCT significantly reduced the risk of grade 3 to 4 aGVHD but did not increase the survival owing to the increased risk of infection [20]. In a previous study by Kim et al. [21], low-dose ATG at $2.5 \mathrm{mg} / \mathrm{kg}$ showed clinical advantage in prevention of moderate to severe aGVHD in mismatched unrelated HSCT. In addition, low-dose ATG has been reported to improve survival outcomes without the risk of serious infection, and to lower the incidence of aGVHD [22]. In a previous study conducted on mismatched unrelated donor HSCT using $7.5 \mathrm{mg} / \mathrm{kg}$ ATG, Pidala et al. [23] reported a lower incidence of severe cGVHD. The CIR of moderate to severe cGVHD was $19 \%$ ( $95 \%$ CI, 10 to 36 ) after 1 year and $28 \%$ (95\% CI, 16 to 48 ) after 2 years. OS was 55\% (95\% CI, 39 to 71 ) after 1 year and 45\% (95\% CI, 27 to 63 ) after 2 years [23]. In a study conducted by the European Group for Blood and Marrow Transplantation (EBMT), patients with AML received allogeneic HSCT from a matched related donor in the CRi state and were given less than $6 \mathrm{mg} / \mathrm{kg}$ of ATG; it was reported that the cGVHD incidence was reduced without an increase in the risk of relapse [24]. Devillier et al. [25] also reported that the ATG dose was a determinant factor associated with relapse and GVHD. However, the dose of ATG used according to the type of transplant and patient were not 
determined and individual approaches were needed [26]. The results of this study suggested that low to medium dose of ATG (2.5 to $7.5 \mathrm{mg} / \mathrm{kg}$ ) would be adequate for better GVHD prophylaxis and better survival outcomes in mismatched HSCT for acute leukemia and MDS. Our study was a multicenter retrospective study involving a relatively large number of patients who underwent mismatched HSCT in eight centers of Korea. Our data would be helpful for selecting the optimal dose of ATG in HLA mismatched HSCT.

However, our study has several limitations. Because our study was a retrospective study, the patient numbers included in each three ATG dose groups were unevenly distributed by physicians discretions and patient population was relatively heterogenous. According to the ATG dose, subsequent infection risk such as by cytomegalovirus and Epstein-Barr virus need to be investigated in further study.

In conclusion, low to medium doses of ATG may be associated with improving survival outcomes and reducing incidence of ecGVHD without increasing the risk of relapse in patients with acute leukemia or MDS undergoing non-TBI-based HLA-mismatched allogeneic HSCT. In the future, we need to investigate precise optimal dose of ATG in HLA-mismatched allogeneic HSCT by performing well-designed prospective clinical studies.

\section{KEY MESSAGE}

1. Optimal dose of anti-thymocyte globulin (ATG) is undetermined in human leukocyte antigen mismatched hematopoietic stem cell transplantation.

2. Low to medium dose of ATG (2.5 to $7.5 \mathrm{mg} / \mathrm{kg}$ ) was associated with longer overall survival and lower incidence rates of extensive chronic graftversus-host disease.

3. Low to medium dose of ATG did not increase the risk of relapse.

\section{Conflict of interest}

No potential conflict of interest relevant to this article was reported.

\section{Acknowledgments}

This work was funded by Ulsan University Hospital (Biomedical Research Center Promotion Fund [17-01]). This research was supported by the Basic Science Research Program through the National Research Foundation of Korea (NRF), funded by the Ministry of Education, Science and Technology (NRF-2017R1C1B5015107). This study was supported in part by Korean National Research Foundation (KNRF) grants 2015M3A9B6073646 and $2017 \mathrm{M}_{3} \mathrm{~A}_{9} \mathrm{G}_{7072564}$ (both to Ho Sup Lee and Jee-Yeong Jeong).

\section{REFERENCES}

1. Korbling M, Przepiorka D, Huh YO, et al. Allogeneic blood stem cell transplantation for refractory leukemia and lymphoma: potential advantage of blood over marrow allografts. Blood 1995;85:1659-1665.

2. Sarantopoulos S. Allogeneic stem-cell transplantation: a T-cell balancing ACT. N Engl J Med 2018;378:480-482.

3. Theurich S, Fischmann H, Chakupurakal G, et al. Anti-thymocyte globulins for post-transplant graft-versushost disease prophylaxis: a systematic review and meta-analysis. Crit Rev Oncol Hematol 2013;88:178-186.

4. Zander AR, Zabelina T, Kroger N, et al. Use of a five-agent GVHD prevention regimen in recipients of unrelated donor marrow. Bone Marrow Transplant 1999;23:889-893.

5. Kroger N, Solano C, Wolschke C, et al. Antilymphocyte globulin for prevention of chronic graft-versus-host disease. N Engl J Med 2016;374:43-53.

6. Remberger M, Ringden O, Hagglund H, et al. A high antithymocyte globulin dose increases the risk of relapse after reduced intensity conditioning HSCT with unrelated donors. Clin Transplant 2013;27:E368-E374.

7. Ayuk F, Diyachenko G, Zabelina T, et al. Comparison of two doses of antithymocyte globulin in patients undergoing matched unrelated donor allogeneic stem cell transplantation. Biol Blood Marrow Transplant 2008;14:913919.

8. Remberger M, Svahn BM, Mattsson J, Ringden O. Dose study of thymoglobulin during conditioning for unrelated donor allogeneic stem-cell transplantation. Transplantation 2004;78:122-127.

9. Devillier R, Labopin M, Chevallier P, et al. Impact of antithymocyte globulin doses in reduced intensity condi- 
tioning before allogeneic transplantation from matched sibling donor for patients with acute myeloid leukemia: a report from the acute leukemia working party of European group of Bone Marrow Transplantation. Bone Marrow Transplant 2018;53:431-437.

10. Portier DA, Sabo RT, Roberts CH, et al. Anti-thymocyte globulin for conditioning in matched unrelated donor hematopoietic cell transplantation provides comparable outcomes to matched related donor recipients. Bone Marrow Transplant 2012;47:1513-1519.

11. Cheson BD, Bennett JM, Kopecky KJ, et al. Revised recommendations of the international working group for diagnosis, standardization of response criteria, treatment outcomes, and reporting standards for therapeutic trials in acute myeloid leukemia. J Clin Oncol 2003;21:46424649 .

12. Przepiorka D, Weisdorf D, Martin P, et al. 1994 Consensus conference on acute GVHD grading. Bone Marrow Transplant 1995;15:825-828.

13. Shulman HM, Sullivan KM, Weiden PL, et al. Chronic graft-versus-host syndrome in man. A long-term clinicopathologic study of 20 Seattle patients. Am J Med 1980;69:204-217.

14. Zeiser R, Blazar BR. Pathophysiology of chronic graftversus-host disease and therapeutic targets. N Engl J Med 2017;377:2565-2579.

15. Zeiser R, Blazar BR. Acute graft-versus-host disease: biologic process, prevention, and therapy. N Engl J Med 2017;377:2167-2179.

16. Bacigalupo A, Lamparelli T, Barisione G, et al. Thymoglobulin prevents chronic graft-versus-host disease, chronic lung dysfunction, and late transplant-related mortality: long-term follow-up of a randomized trial in patients undergoing unrelated donor transplantation. Biol Blood Marrow Transplant 2006;12:560-565.

17. Drobyski WR, Ash RC, Casper JT, et al. Effect of T-cell depletion as graft-versus-host disease prophylaxis on engraftment, relapse, and disease-free survival in unrelated marrow transplantation for chronic myelogenous leukemia. Blood 1994;83:1980-1987.

18. Finke J, Bethge WA, Schmoor C, et al. Standard graftversus-host disease prophylaxis with or without anti-Tcell globulin in haematopoietic cell transplantation from matched unrelated donors: a randomised, open-label, multicentre phase 3 trial. Lancet Oncol 2009;10:855-864.

19. Socie G, Schmoor C, Bethge WA, et al. Chronic graftversus-host disease: long-term results from a randomized trial on graft-versus-host disease prophylaxis with or without anti-T-cell globulin ATG-Fresenius. Blood 2011;117:6375-6382.

20. Bacigalupo A, Locatelli F, Lanino E, et al. Fludarabine, cyclophosphamide and anti-thymocyte globulin for alternative donor transplants in acquired severe aplastic anemia: a report from the EBMT-SAA Working Party. Bone Marrow Transplant 2005;36:947-950.

21. Kim HJ, Min WS, Cho BS, et al. Successful prevention of acute graft-versus-host disease using low-dose antithymocyte globulin after mismatched, unrelated, hematopoietic stem cell transplantation for acute myelogenous leukemia. Biol Blood Marrow Transplant 2009;15:704-717.

22. Basara N, Baurmann H, Kolbe K, et al. Antithymocyte globulin for the prevention of graft-versus-host disease after unrelated hematopoietic stem cell transplantation for acute myeloid leukemia: results from the multicenter German cooperative study group. Bone Marrow Transplant 2005;35:1011-1018.

23. Pidala J, Tomblyn M, Nishihori T, et al. ATG prevents severe acute graft-versus-host disease in mismatched unrelated donor hematopoietic cell transplantation. Biol Blood Marrow Transplant 2011;17:1237-1244.

24. Baron F, Labopin M, Blaise D, et al. Impact of in vivo T-cell depletion on outcome of AML patients in first CR given peripheral blood stem cells and reduced-intensity conditioning allo-SCT from a HLA-identical sibling donor: a report from the Acute Leukemia Working Party of the European Group for Blood and Marrow Transplantation. Bone Marrow Transplant 2014;49:389-396.

25. Devillier R, Labopin M, Chevallier P, et al. Higher doses of antithymocyte globulin (ATG) increase the risk of relapse in acute myeloid leukemia (AML) patients undergoing matched related donor allogeneic transplantation in first complete remission (CR1): an analysis from the acute leukemia working party of EBMT. Blood 2014;124:729.

26. Kekre N, Antin JH. ATG in allogeneic stem cell transplantation: standard of care in 2017? Counterpoint. Blood Adv 2017;1:573-576. 